\title{
ONS Data on COVID-19 deaths in children is reliable, and increasingly of concern (Re: "Deaths in children and young people in England after SARS-CoV-2 infection during the first pandemic year")
}

\author{
Tom Lawton ${ }^{1}$, Deepti Gurdasani ${ }^{2}$, Stephen Griffin ${ }^{3}$, and James Neill ${ }^{4}$ \\ ${ }^{1}$ Bradford Institute for Health Research \\ ${ }^{2}$ Queen Mary University of London \\ ${ }^{3}$ University of Leeds \\ ${ }^{4}$ Long Covid Kids
}

November 18, 2021

\begin{abstract}
The recently published "Deaths in children and young people in England after SARS-CoV-2 infection during the first pandemic year" ${ }^{1}$ attempts to unpick the issue of paediatric deaths "with" versus "from" COVID-19, additionally reporting on preexisting comorbidities of the children and young people (CYP) who died after a COVID-19 diagnosis. Linking data from the National Child Mortality Database (NCMD), hospital data from the Secondary Uses Service (SUS), and PICU audit data, the authors have re-examined deaths in CYP after a COVID-19 diagnosis using these datasets. However, whilst data on any pre-existing conditions may be useful to identify the children at highest risk from COVID-19, we have some concerns around the methodology and presentation of the first part of this study.
\end{abstract}

First, the justification of the methodology - using a review of secondary datasets to determine primary and contributing causes of death - is unclear, given that equivalent death certification data from the Office for National Statistics (ONS) has been available throughout the study period. Given ONS data involves death certification, usually by the treating physician, with access to full case records rather than the more limited data in the databases used in the study, it is unclear why the authors chose to re-review causes of death based upon more limited data stemming from deaths after a positive COVID-19 test. Indeed, the article does not mention mortality data from the ONS at all, whichprovides eminently comparable estimates for 0-17 year olds during the chosen interval. ${ }^{2}$ Indeed, from the same period ONS data shows 20 deaths in 0-17 year olds (England and Wales) compared to 25 in the study (England). We note, however, that PIMS-TS (Paediatric inflammatory multisystem syndrome temporally associated with SARS-CoV-2) data may not have been recorded accurately within the ONS due to absence of relevant codes; the study therefore adds value by reviewing potential fatalities associated with cases of PIMS-TS based on other codes.

While the authors have extensively discussed the role of pre-existing conditions as contributing to deaths, it is unclear how this was determined, and why again ONS death certification data was not used directly to assess this, given it lists both direct and contributing factors. ${ }^{3}$ The process involved with attributing the contribution to death by pre-existing conditions also remains unclear, and it is notable that there was significant disagreement between reviewers; all three reviewers only agreed on half the cases reviewed, and $20 \%$ (9 of 44) of these were considered unclassifiable based upon the methods used. ONS reports that, during the study period, 85\% of CYP fatalities involving COVID-19 had it as the primary underlying cause 
of death. $^{2}$

A clear inference from Smith et al. should be that ONS data is, as expected, extremely reliable. This brings us to the second major limitation of this work, which is the lack of generalisability to the current context. The study was carried out during a period where there were significant community mitigations, long periods of school closures, and before the more transmissible ${ }^{4}$ delta variant became dominant. Since the study period, recorded cases in CYP have been over three times higher, ${ }^{5}$ and ONS reports 73 deaths involving COVID-19 in 0-19 year olds up to $5^{\text {th }}$ November $2021^{6}$ (with 42 deaths in 0-17 year olds up to September $2021,{ }^{2}$ more recent data not yet being available). Furthermore, data following the study period (Mar-Sep 2021) shows that over $93 \%$ of all deaths in children "within" 28 days of a positive SARS-Cov-2 test are subsequently confirmed by the ONS as deaths directly involving COVID-19., ${ }^{5,6}$ This means that one of the key messages of the paper, namely that only $41 \%$ of deaths recorded by PHE "with" COVID-19 are "from" COVID-19, is no longer valid. Indeed, given the lags in death certification, recent deaths are currently best estimated using UKHSA 28-day death data (Figure 1). Both ONS and UKHSA data suggest that deaths in CYP are now the highest they have ever been during the pandemic. This is a predictable consequence of removing the majority of mitigations in schools, along with a delayed and now lagging vaccination programme in CYP. ${ }^{7}$ The resultant mass infection of children means that, despite the relative rarity compared to adults of severe outcomes at the individual level, an unacceptable number of preventable deaths has occurred in CYP in the latter part of 2021. Fortunately CYP rarely die of anything: in Jun-Sep 2021 ONS reported a total of 446 deaths of 5-19 year olds from any cause, however $4.3 \%$ of those deaths [n=19] had COVID-19 as the underlying cause. COVID-19 is a significant cause of death for CYP. ${ }^{2}$

Thirdly, we are concerned by the use of the whole CYP population as a denominator for the headline mortality figure of 2 per million population, which is compared with all-cause deaths in children. This is at odds with recommended methodology, ${ }^{8}$ and misleading given that deaths due to COVID-19 in children are dependent on exposure rather than population size. Exposure is a result of disease prevalence and the effects of mitigations, and so has changed considerably at different points during the pandemic. Indeed, it has been much higher following the introduction of the delta variant into the UK, coincident with the removal of most mitigations; this likely explains the much higher number of deaths since the study completed. If we were to calculate a population rate at the present time (which would again be inappropriate) it would be 2-3 times the rate reported in this study. Moreover, as deaths accrue in children, the population rate will continue to rise. ${ }^{9}$

The authors report an estimated infection fatality rate (IFR) of 5 per 100,000, based on PHE modelling of the number of infections in children. They clarify that they did not use the number of positive SARS-CoV-2 tests as a denominator as these have likely underestimated infections, particularly during the first wave, as well as re-infections in children. However, they also state that "99.995\% [...] with a positive SARS-CoV-2 test survived". Not only is this way of reporting risk not in line with standard epidemiological practice,${ }^{10}$ it also appears to directly contradict the authors' stated methodology by suggesting a case fatality rate (CFR) rather than an IFR; and the flaws in the numerical approach are highlighted by it being adopted by certain groups seeking to minimise the impact of this disease by promoting misinformation. ${ }^{11}$ The methodology for derivation of this rate is also unclear, as the provided reference does not point to a modelling estimate by PHE (as stated in the text) or methodology for how these cases were estimated. Making a distinction between a CFR and IFR is important, given infections in children are often vastly underestimated by routine testing. Unfortunately, this error has already been replicated by the popular press. ${ }^{12,13}$

Whilst this IFR may appear to be low, it is widely anticipated that without mitigations and with most CYP remaining unvaccinated (or partially vaccinated), almost all children will be infected with SARS-CoV2 at least once. Considering Smith et al.'s estimated IFR, this could potentially lead to 640 deaths "from" COVID-19 amongst the $>12$ million English CYP population. This compares extremely poorly to seasonal influenza, where there were 13 and 25 deaths of CYP related to influenza in the 2018-19 and 2019-20 periods, respectively. ${ }^{14,15}$ Prior to the Oct 2021 half-term at the peak of infection, the prevalence of infection was over $5 \%$ in a single week in primary and secondary school age children, ${ }^{16}$ with approximately 200,000 confirmed 
cases in CYP in just a two week period. ${ }^{5}$

In summary, by utilising less comprehensive data sets and reassessing COVID-related CYP fatalities, Smith et al. in fact somewhat predictably validate ONS data on CYP deaths as robust and reliable. By extension, it is clear that removal of most mitigations from schools and delays in vaccination have led to unacceptably high levels of deaths among children, which continue to increase (Figure 1). UK schools (Department for Education) and vaccination (Joint Committee for Vaccination and Immunisation) policies for children are outliers across the globe, and this has led directly to an extremely high rate of SARS-CoV2 exposure in CYP. Of considerable yet similarly predictable concern, ONS data shows substantial increases in self-reported long COVID incidence amongst children in recent months. ${ }^{17}$ Urgent action is needed to protect children from this preventable disease.

\section{Figure}

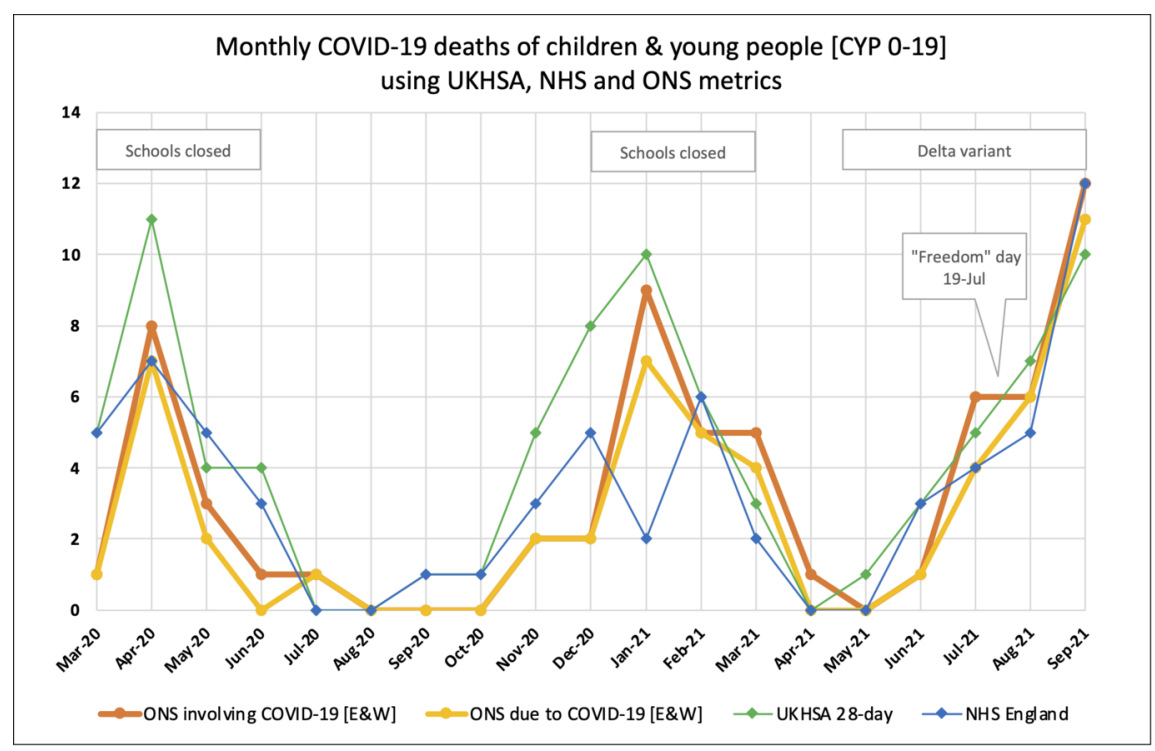

Figure 1: Monthly deaths in CYP as measured by four different metrics

\section{References}

[1] Smith C, Odd D, Harwood R, Ward J, Linney M, Clark M, et al. Deaths in children and young people in England after SARS-CoV-2 infection during the first pandemic year. Nat Med 2021:1-8. https://doi.org/10.1038/s41591-021-01578-1.

[2]

Office for National Statistics. Single year of age and average age of death of people whose death was due to or involved COVID-19 2021. https://www . ons.gov.uk/peoplepopulationandcommunity/birthsdeathsandmarriages/deaths/datasets/ singleyearof ageandaverageageof deathof peoplewhosedeathwasduetoorinvolvedcovid19 (accessed November 12, 2021).

[3] HM Passport Office. Guidance for doctors completing Medical Certificates of Cause of Death in England and Wales 2020. 
[4] Twohig KA, Nyberg T, Zaidi A, Thelwall S, Sinnathamby MA, Aliabadi S, et al. Hospital admission and emergency care attendance risk for SARS-CoV-2 delta (B.1.617.2) compared with alpha (B.1.1.7) variants of concern: a cohort study. Lancet Infect Dis 2021;0. https://doi.org/10.1016/S14733099(21)00475-8.

[5] UK Health Security Agency. Coronavirus in the UK n.d. https://coronavirus.data.gov . uk/ (accessed November 12, 2021).

[6] Office for National Statistics. Deaths registered weekly in England and Wales by age and sex 2021. https://www.ons.gov.uk/datasets/weekly-deaths-age-sex/editions/covid-19/versions/58 (accessed November 18, 2021).

[7] Alwan NA. We must call out childism in covid-19 policies. BMJ 2021;375:n2641. https://doi.org/10.1136/bmj.n2641.

[8] Estimating mortality from COVID-19. World Health Organization; 2020. Report No.: WHO2019-nCoV-Sci_Brief-Mortality-2020.1.

[9] Gianicolo E, Riccetti N, Blettner M, Karch A. Epidemiological Measures in the Context of the COVID-19 Pandemic. Dtsch Ärztebl Int 2020. https://doi.org/10.3238/arztebl.2020.0336.

[10] Dicker R, Coronado F, Koo D, Parrish RG. Principles of Epidemiology in Public Health Practice, 3rd Edition. 2011.

[11] Long-touted COVID-19 "survival rate" figure is overdue to expire. Aust Assoc Press 2021. https://www.aap.com.au/factcheck/long-touted-covid-19-survival-rate-figure-isoverdue-to-expire/ (accessed November 12, 2021).

[12] Pinkstone J. Just six healthy children died of Covid in a year, NHS analysis shows. The Telegraph 2021.

[13] Senior JE. Only SIX healthy children died of Covid in first year of the pandemic. Mail Online 2021.

[14] Surveillance of influenza and other respiratory viruses in the UK Winter 2018 to 2019. Public Health England; 2019.

[15] Surveillance of influenza and other respiratory viruses in the UK Winter 2019 to 2020. Public Health England; 2020.

[16] Chadeau-Hyam M, Eales O, Bodinier B, Wang H, Haw D, Whitaker M, et al. REACT-1 round 15 interim report: High and rising prevalence of SARS-CoV-2 infection in England from end of September 2021 followed by a fall in late October 2021. Epidemiology; 2021. https://doi.org/10.1101/2021.11.03.21265877.

[17] Prevalence of ongoing symptoms following coronavirus (COVID-19) infection in the UK: 4 November 2021. Office for National Statistics; 2021. 